\title{
Chemochemical caries removal: a review of the techniques and latest developments
}

\author{
J. A. Beeley, ${ }^{1}$ H. K. Yip, ${ }^{2}$ and A. G. Stevenson, ${ }^{3}$
}

Chemomechanical caries removal involves the chemical softening of carious dentine followed by its removal by gentle excavation. The reagent involved is generated by mixing amino acids with sodium hypochlorite; $\mathbf{N}$-monochloroamino acids are formed which selectively degrade demineralised collagen in carious dentine. The procedure requires 5-15 minutes but avoids the painful removal of sound dentine thereby reducing the need for local anaesthesia. It is well suited to the treatment of deciduous teeth, dental phobics and medically compromised patients. The dentine surface formed is highly irregular and well suited to bonding with composite resin or glass ionomer. When complete caries removal is achieved, the dentine remaining is sound and properly mineralised. The system was originally marketed in the USA in the 1980's as Caridex. Large volumes of solution and a special applicator system were required. A new system, Carisolv, has recently been launched on to the market. This comes as a gel, requires volumes of $0.2-1.0 \mathrm{ml}$ and is accompanied by specially designed instruments.

\begin{abstract}
The earliest attempts to remove caries 1 involved the use of a hand drill which was soon surpassed in 1871 by James Morrison's treadle instrument developed from the mechanisms of Isaac Singer's sewing machine. Modern high speed drills are the latest development of this more than a century old technique. ${ }^{1}$ Over the years, other procedures have also been used for caries removal (CMCR). These include air abrasion with aluminium oxide, chemomechanical caries removal, atraumatic restorative therapy $(\mathrm{ART})^{2}$ and most recently, lasers. ${ }^{3}$ With the recent launch onto the market of a new product for CMCR, there is renewed interest in this procedure which selectively removes carious dentine but avoids the painful and unnecessary removal of sound dentine. Restoration of cavities prepared by this technique requires materials such as
\end{abstract}

1Senior Lecturer in Oral Biochemistry, 3 Lecturer in Adult Dental Care, University of Glasgow Dental School, 378 Sauchiehall St, Glasgow, G2 3JZ. 2Assistant Professor, Faculty of Dentistry, The Prince Philip Dental Hospital, Hong Kong SAR, China.. Correspondence to J. A. Beeley

REFEREED PAPER

Received 17.12.1998; ACCEPTED 24.09.1999

(C) British Dental Journal 2000; 188: 427-430 composite resins or glass ionomers which bond to the dentine surface rather than materials such as amalgams which involve cutting a cavity designed to mechanically retain the restoration.

Dentine consists of mineral (70\%wt.), water $(10 \% \mathrm{wt}$,) and an organic matrix (20\%wt.). Of this organic matrix, $18 \%$ is collagen and $2 \%$ non-collagenous compounds including chondroitin sulphate, other proteoglycans and phosphophoryns. ${ }^{4-5}$ Collagen is an unusual protein which contains large amounts of proline and one third of the amino acid content is glycine. The polypeptide chains are coiled into triple helices which are known as tropocollagen units; these tropocollagen units then orientate side by side to form a fibril. Co-valent bonds between the polypeptide chains and between the tropocollagen units form cross links and give the collagen fibrils stability (Fig. 1), in dentine the fibrils are in the form of a dense meshwork which becomes mineralised. ${ }^{6}$

When caries occurs, acids produced by plaque bacteria by anaerobic fermentation of carbohydrate initially cause solubilisation of the mineral in enamel. As the process
In brief

- Chemochemical caries removal involves the selective removal of carious dentine. The reagent is prepared by mixing solutions of amino acids and sodium hydrochlorite.

- Reagents for the procedure were originally marketed as a solution known as Caridex. More recently, a similar system in the form of a gel has been marketed as Carisolv.

- Being a gel, Carisolv has the advantage of requiring volumes of less than $1 \mathrm{ml}$. No applicator system other than specially designed instruments is required.

- Because only carious dentine is removed, the painful removal of sound dentine is avoided and the need for local anaesthesia is minimised.

- The procedure is suitable for soft carious lesions access to which may still require conventional mechanical procedures.

progresses, dentinal tubules provide access for penetrating acids and subsequent invasion by bacteria which results in a decrease in $\mathrm{pH}$ and causes further acid attack and demineralisation. When the organic matrix has been demineralised, the collagen and other matrix components are then susceptible to enzymatic degradation, mainly by bacterial proteases and other hydrolases. ${ }^{7}$ With respect to collagen degradation, two zones can usually be distinguished within a lesion. There is an inner layer which is partially demineralised and can be remineralised and in which the collagen fibrils are still intact, and there is an outer layer where the collagen fibrils are partially degraded and cannot be remineralised. ${ }^{8}$ A CMCR reagent must be able to cause further degradation of this partially degraded collagen, by cleavage of the polypeptide chains in the triple helix and/or hydrolysing the cross linkages as explained in Figure 1.

The principal on which CMCR is based arises from studies by Goldman and Kronman working in New Jersey, U.S. in the 1970's. They were studying the effect of 


\section{PRACTICE conservative dentistry}

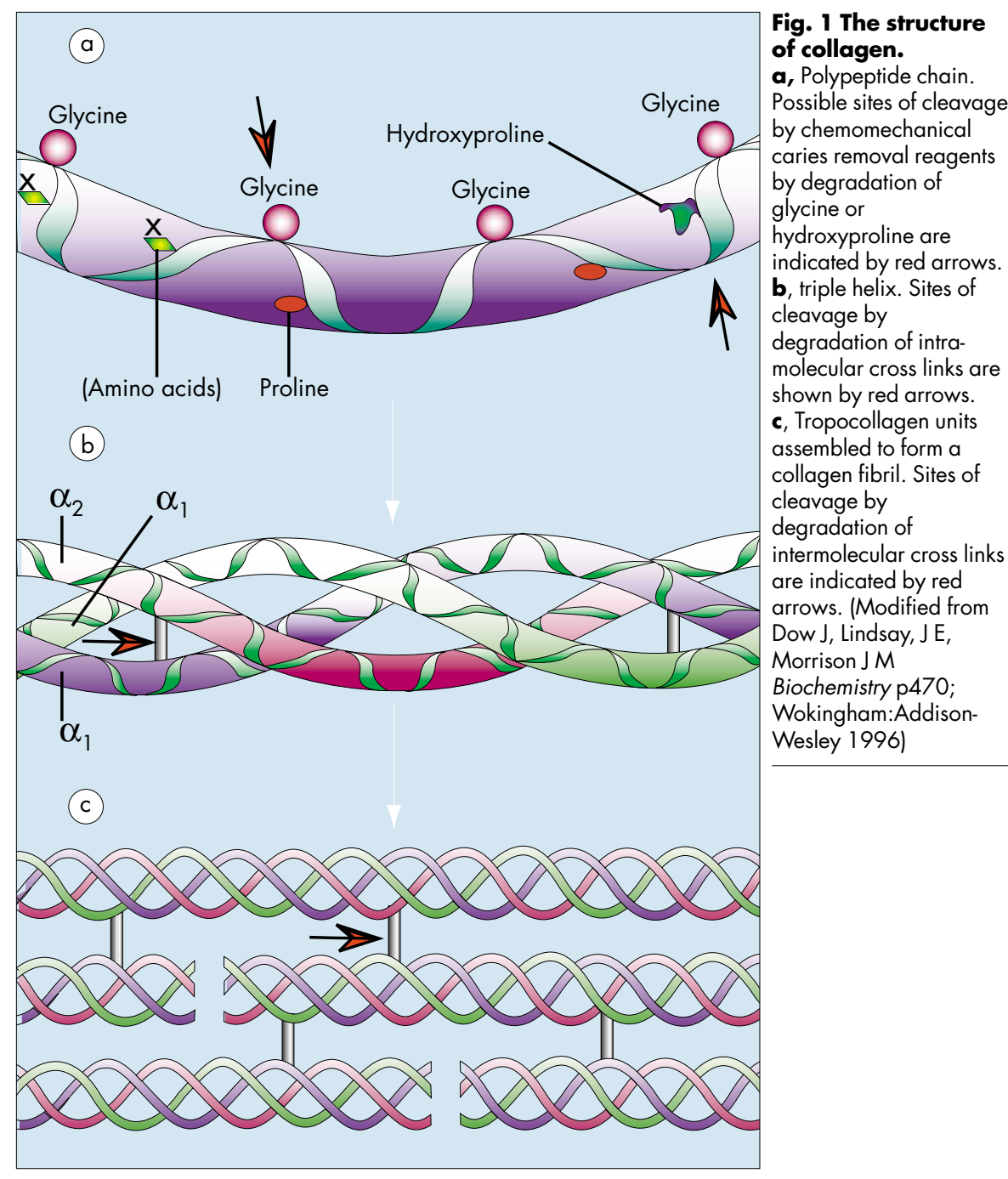

sodium hypochlorite, which is a non-specific proteolytic agent, on the removal of carious material from dentine. Sodium hypochlorite itself however was too corrosive for use on healthy tissue and so they decided to incorporate it into Sorensen's buffer (which contains glycine, sodium chloride and sodium hydroxide) in an attempt to minimise this problem. Quite fortuitously a reaction occurred which resulted in a product which was more effective in removal of carious dentine than a saline placebo. This involved the chlorination of glycine to form N-monochloroglycine (NMG) and the reagent subsequently became known as GK-1019.9 In subsequent studies they found that the system was more effective if glycine was replaced by amino butyric acid, the product then being N-monochloroaminobutyric acid (NMAB) also designated GK-101E. ${ }^{10}$

The mechanism of action of NMG and NMAB on collagen is still unclear and knowledge of the chemistry of chlorination of amino acids and their effects is still very limited. Originally it was thought that the procedure involved chlorination of the partially degraded collagen in the carious lesion and the conversion of hydroxyproline to pyrrole-2-carboxylic acid. ${ }^{11}$ More recent work suggests that cleavage by oxidation of glycine residues could also be involved. ${ }^{12}$ This causes disruption of the collagen fibrils which become more friable and can then be removed.

The NMAB system was patented in the US in 1975 and a further patent taken out by the National Patent Dental Corporation, New York in 1987. It received FDA approval for use in the USA in 1984 and was marketed in the 1980's as Caridex. ${ }^{13}$ It consisted ot two solutions, Solution I containing sodium hypochlorite and Solution II containing glycine, aminobutyric acid, sodium chloride and sodium hydroxide. The two solutions were mixed immediately before use to give the working reagent ( $\mathrm{pH}$ approx. 11) which was stable for one hour.

A delivery system was also available which consisted of a reservoir for the solution, a heater and a pump which passed the liquid warmed to body temperature through a tube to a hand piece and an applicator tip which came in various shapes and sizes. The solution was applied to the carious lesion by means of this applicator which was used to loosen the carious dentine by a gentle scrap- ing action; the debris together with the spent solution being removed by aspiration. Application was continued until the dentine remaining was deemed sound by normal clinical tactile criteria. With suitable accessible soft lesions, after 5-10 minutes treatment only clinically sound dentine remained.

The reagent selectively removed carious dentine leaving a surface with many overhangs and undercuts; dentine 'scales' were a frequent feature of the surfaces formed and dentinal tubules were both patent and occluded (Fig. 2). The surface would appear to be the interface between carious and sound dentine. ${ }^{14}$ This surface should be well suited to restoration with modern adhesive materials such as glass ionomers as the bond formed with these materials has been shown to be stronger than with a conventional smear layer. ${ }^{15}$

The procedure avoids the painful removal of sound dentine but is ineffective in the removal of hard eburnated parts of the lesion; removal of eburnated caries however may not be necessary. ${ }^{16}$ Recently it has been shown that discolouration in carious dentine results from the Maillard reaction which modifies amino acids in collagen thereby making them more resistant to proteolytic attack and inhibiting lesion progression in discoloured dentine. ${ }^{17,18}$

Toxicity studies have shown the solution to be safe and to have no adverse effects on pulp or healthy tissue. Although a few patients find the taste unpleasant, generally this is not a problem and patient acceptance is high. Its advantages include reduced need for local anaesthesia, conservation of sound tooth structure and reduced risk of pulp exposure. It is well suited to the treatment of anxious or medically compromised patients as well as to paediatric and domiciallary dentistry.

There are however some limitations with the use of this system. Rotary and/or hand instruments may still be needed for the removal of tissue or material other than degraded dentine collagen. This includes access to small or interproximal carious lesions, removal of enamel overlying the caries, removal of existing restorations, etc. as well as for cavity design when non-adhesive restorative materials are used.

Although the Caridex system initially 


\section{PRACTICE conservative dentistry}

proved to be quite popular, large volumes of solution were needed $(200-500 \mathrm{ml})$ and the procedure was slow. Only certain cavities were suitable for treatment by the technique and because of the time involved (10-15 mins) and limited use, popularity in the US waned. Although there were studies on the efficacy of caries removal by the procedure, studies on the long term success of cavities restored after CMCR treatment were lacking. In the early 1990's Caridex ceased to be marketed and the manufacturer's patent lapsed.

Interest in the UK began in the late 1980s in Glasgow and London. Whilst the London groups at UMDS Guy's Campus (now the GKT Dental Institute) ${ }^{19}$ and the 'London' (now the St. Bartholomews and Royal London Hospital School of Medicine and Dentistry) ${ }^{13}$ carried out some more clinical studies together with studies on bonding to adhesives ${ }^{14}$ the group in Glasgow attempted to modify the formulation and develop an improved CMCR reagent. In vitro studies were carried out on permanent and deciduous teeth using an NMAB solution similar in composition to Caridex. They found that CMCR was more effective on deciduous teeth than on permanent ones. An attempt was made to improve the reagent by the addition of urea, which normally denatures proteins by breaking down hydrogen bonding thereby making them more soluble. Although urea itself was no better than a saline control as a CMCR reagent, addition of urea to NMAB improved the efficacy of the formulation. Indeed, NMAB was only statistically significantly more effective than a saline control

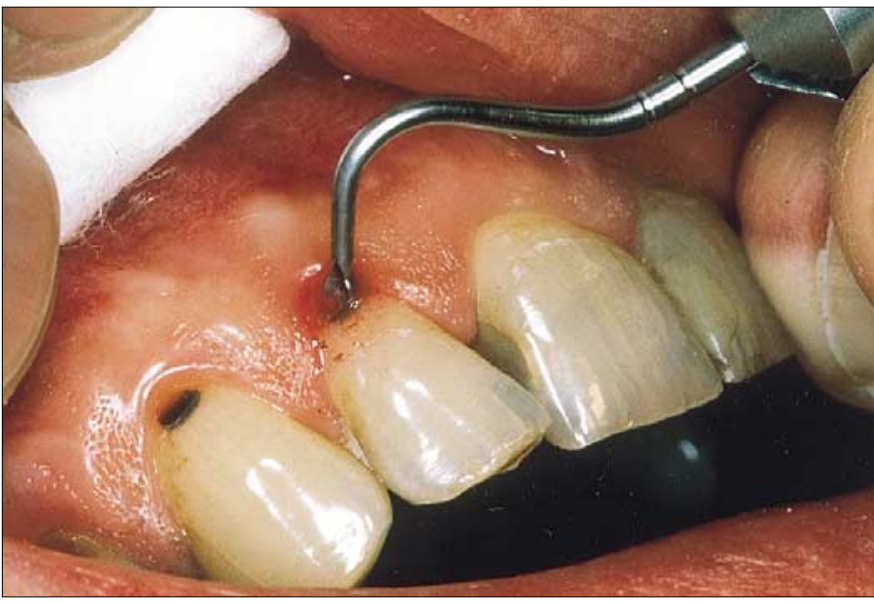

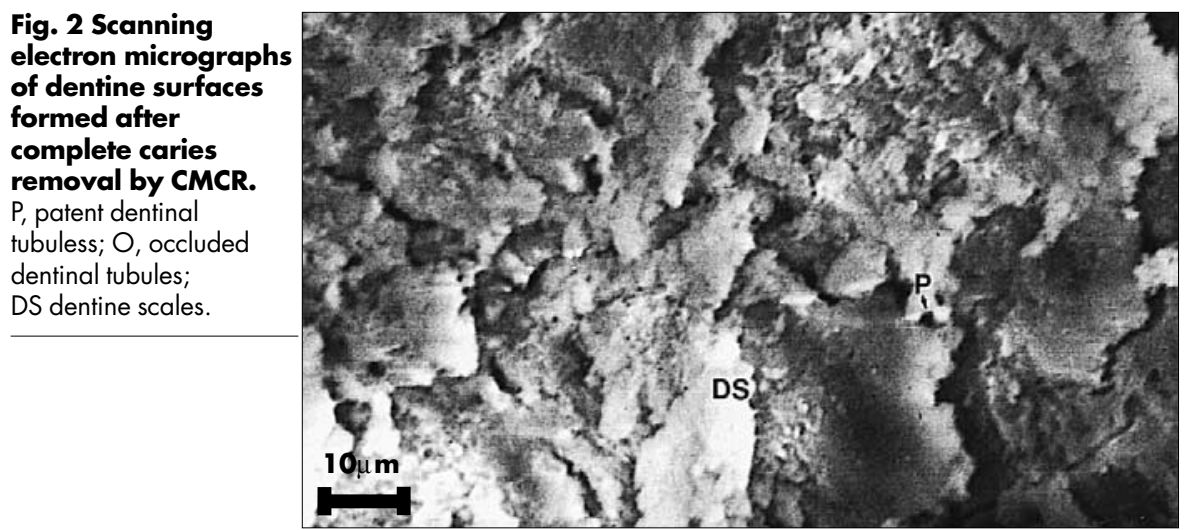

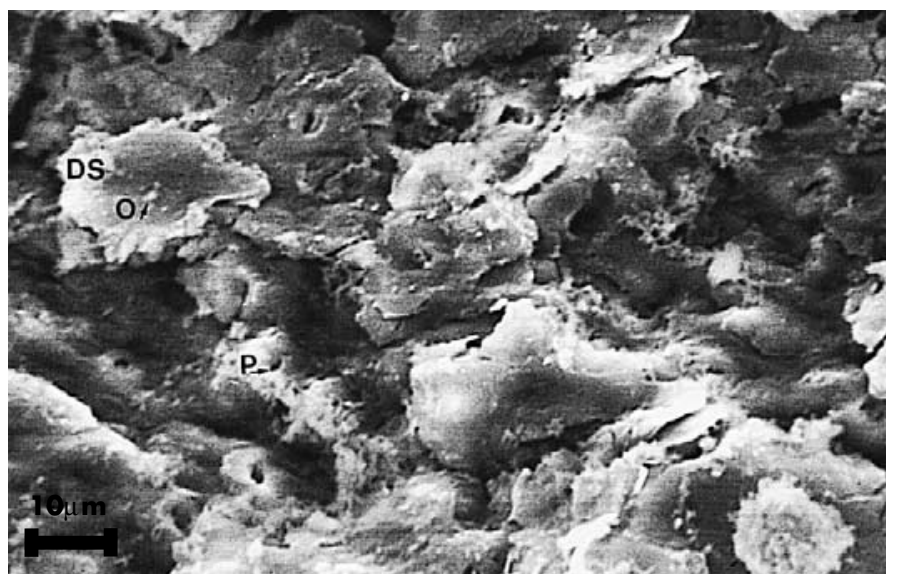

when it contained urea, ${ }^{20}$ a finding which has been confirmed in a subsequent more carefully controlled study. ${ }^{21}$ Other workers have found that in vitro there was no difference between the efficacy of NMAB and water in terms of ease of caries removal by the chemomechanical procedure. ${ }^{22}$

Detailed studies have been carried out on the nature of the dentine surface remaining after complete caries removal by CMCR. Electron probe micro-analysis showed that the dentine is sound and properly mineralised and that the surface formed is highly irregular. ${ }^{23}$ Histological studies have confirmed the irregular nature of the dentine surface and also shown that some dentinal tubules contain bacteria but the level of these is no higher than in mechanically pre- pared cavities. $^{21}$

There have been several reports of clinical trials involving Caridex and similar solutions. These have included all classes of coronal lesions, cervical lesions and root caries and involved both deciduous and permanent teeth. The reported number of teeth in which complete removal of carious dentine has been achieved ranges from $42-100 \%$ with the majority showing values of $80 \%$ or more; the results have been reviewed elsewhere. ${ }^{20}$ These trials were generally not well designed however and there is little information about the long term success of lesions restored after CMCR treatment. A 3-year follow-up study is currently taking place in Glasgow comparing laser and CMCR removal of carious dentine with conventional treatment carried out under local and general anaesthesia.

Because of the time required for CMCR treatment, the large volumes of solution needed and the fact that the delivery system was no longer commercially available, use of CMCR, despite its potential, became minimal.

During this time however, Medi Team in Sweden continued to work on the system and the latest CMCR reagent known as Carisolv hit the headlines in January 1998. Although this is similar to the Caridex and NMAB systems, it is in the form of a pink gel which can be applied to the carious lesion with specially designed hand instruments 


\section{PRACTICE conservative dentistry}

\begin{tabular}{|c|c|c|}
\hline \multirow[t]{2}{*}{ Table 1} & \multicolumn{2}{|c|}{ Comparison of Coridex and Corrisolv } \\
\hline & CARIDEX & CARISOLV \\
\hline Solution 1 & $1 \% \mathrm{NaOCl}$ & $0.5 \% \mathrm{NaOCl}$ \\
\hline Solution 11 & $\begin{array}{l}0.1 \mathrm{M} \text { aminobutyric acid glycine } \\
0.1 \mathrm{M} \mathrm{NaCl} \\
0.1 \mathrm{M} \mathrm{NaOH}\end{array}$ & $\begin{array}{l}0.1 \mathrm{M} \text { glutamic acid/leucine/lysine } \\
\mathrm{NaCl}^{*} \\
\mathrm{NaOH}^{*}\end{array}$ \\
\hline Dye & - & Erythrocin (pink) \\
\hline $\mathrm{pH}$ & 11 & 11 \\
\hline Physical properties & Liquid & Gel \\
\hline Volume Needed & $100-500 \mathrm{ml}$ & $0.2-1.0 \mathrm{ml}$ \\
\hline Time required & $5-15$ mins & $5-15$ mins \\
\hline Equipment Required & Applicator Unit & None \\
\hline Instruments & Applicator tips & Specially designed \\
\hline $\begin{array}{l}\text { Time preparation } \\
\text { Remains active } \\
\text { after mixing }\end{array}$ & 1 hour & 20 mins \\
\hline
\end{tabular}

which have recently been modified. ${ }^{24}$ Because it is a gel, the volume required is now less than one millilitre and it requires neither heating nor a delivery system. It is marketed in two syringes, one containing the sodium hypochlorite solution and the other a pink viscous gel which contains three amino acids, lysine, leucine and glutamic acid, together with carboxymethylcellulose to make it viscous and erythrocin to make it readily visible in use. The contents of the two syringes are mixed by a simple system which involves joining the two together immediately before use as its effectiveness begins to deteriorate after $20 \mathrm{~min}$ utes. More recently a new twin syringe mixing system containing sufficient material for 10-15 treatments has been introduced. This dispenses the exact amount required through a disposable mixing tip, and it can be active for up to one month if stored in a refrigerator after opening.

The gel is applied to the carious lesion with one of the hand instruments and after 30 seconds, carious dentine can be gently removed (Fig 3). More gel is then applied and the procedure repeated until no more carious dentine remains, a guide to this being when the gel removed from the tooth is clear. The time required for the procedure is about 9-12 minutes (range about 5-15 minutes) and the volume of gel is only $0.2-1.0 \mathrm{ml} .{ }^{24}$ Rotary instruments may still be required however for some cavities but preliminary reports indicate that patient acceptance is very good. The system is much easier to use than Caridex and, because it involves a gel rather than a liquid, there is better contact with the carious lesion. When complete caries removal is achieved by this technique, the cavity surface has been shown to be as sound as that remaining after conventional drilling. ${ }^{25,26}$ Reports of independent clinical trials on the use of the sys- tem in practice and on the long term success of the treatment however are still awaited. Hopefully statistical analysis of the findings will show it to be more effective than Caridex. Caridex and Carisolv are compared in Table 1.

Development of a clinically effective caries removal reagent which is harmless to healthy tissue and bio-compatible to the pulp is fundamentally difficult because the molecular structures of these tissues are so similar. Whilst CMCR necessitates a selective reagent which will further degrade and partially solubilise partially degraded dentine collagen, soft tissue collagen present in the pulp and surrounding tissues is similar in structure. The new system offers considerable attractions in certain cases, but if such a system is to become routinely used it may need to be still more rapid in its mode of action.

1 Ring M E. Dentistry; an Illustrated History. pp 250-251.New York: Abrams, 1985.

2 Frencken J E, Pilot T, Sangpaison Y Phantumvanit P. Atraumatic restorative treatment (ART): rationale technique and development J Public Health Dent 1996; 56: 135-140.

3 Keller U Hibst R, Guertsen W, Schilke R, Heidermann D, Klacber B, Raab W H M. Erbium : YAG laser applications in caries therapy: evaluation of patients perception and acceptance. J Dent 1998: 26; 649-656.

4 Veis A. In Comper W.D. (Ed) Extracellular Matrix, Volume 1 Tissue Function. 41-76 Amsterdam: Harwood Academic Press, 1996.

5 Hall R C, Embery G. The use of immunohistochemistry in understanding the structure and function of the extracellular matrix of dental tissues. Adv Dent Res 1997; 11: 478-486.

6 Van der Rest M, Bruckner P. Collagens: diversity at the molecular and super molecular levels. Curr Opin Struct Biol 1993; 3: 430-436

7 Thylstrup A, Fejerskov O. Text book of Clinical Cariology. (2nd ed) 111-157. Copenhagan: Munksgaard 1994.

8 Ogushi K, Fusayama T. Electron microscopic structures of two layers of carious dentine. Dent Res 1975; 54: 1019-1026.

9 Goldman M, Kronman J H. A preliminary report on a chemomechanical means of removing caries J Amer Dent Assoc 1976; 93:
1149-1153.

10 Schutzbank S G, Marchwinski M, Kronman J $\mathrm{H}$, Goldman M, Clark R E. A comparative in vitro study of GK-101 and GK-101E in caries removal J Dent Res 1978; 57: 861-864

11 Habib C M, Kronman J, Goldman M. A chemical evaluation of collagen and hydroxyproline after treatment with GK-101 (N-monochloroglycine). Phar Thera Dent 1975; 2; 209-215.

12 Yip H K, Beeley J A. Studies on the reaction of NaOCI and NMAB with collagen J. Dent Res 1989; 68: 982.

13 Burke F, Lynch E. Chemomechanical caries removal. J Irish Dent Assoc 1995: 41; 10-14

14 Yip H K, Beeley J A, Stevenson A G. The interface between carious and sound dentine an SEM study. Med Sci Res 1991: 19; 187-188.

15 Burke F M, Lynch E. The effect of chemomechanical caries removal on the bond strength of glass polyalkenoate cement to dentine. $J$ Dent 1994: 22; 283-291.

16 Kidd E A M, Joyston-Bechel S, Beighton D. Microbiological validation of assessments of caries activity during cavity preparation. Caries Res: 27; 402-408.

17. Lynch E, Sheerin A, Silwood C J, Grootveld M C. Detection and determination of protein carbonyl adducts in primary root caries. $J$ Dent Res: 76; 1049.

18 Kleter G A, Damen J J M, Buigus M J, ten Cate J M. Modification of amino acid residues in carious dentine matrix. J Dent Res 1998: 77; 488-495.

19 Watson T F, Kidd E A M. USA: the Caridex caries removal system. Br Dent J 1986: 161; 461-462.

20 Yip H K, Stevenson A G, Beeley J A. An improved reagent for chemomechanical removal of dental caries in permanent and deciduous teeth. An in vitro study J. Dent 1995; 23: 197-204.

21 Yip H K, Stevenson A G, Beeley J A. Chemomechanical removal of dental caries in deciduous teeth: further studies in vitro $\mathrm{Br}$ Dent J 1999; 186: 179-182.

22 Bawart O, Moschen I, Graber A, Pfaller K. In vitro study comparing the efficacy of $\mathrm{N}$ monochloro-D, L-2aminobutyrate (NMAB, GK-101E) and water in caries removal. J Oral Rehabil 1991: 18; 535-536.

23 Yip H K, Beeley J A, Stevenson A G. Mineral content of the dentine remaining after chemomechanical caries removal. Caries Res 1995; 29: 111-117.

24 Ericson D, Simmerman M, Raber H, Götrick $\mathrm{B}$, Bornstein R. Clinical evaluation of efficacy and safety of a new method for chemomechanical removal of caries. Caries Res 1999; 33: 171-177.

25. Moran C, Lynch E, Petersson L, Borshboom P. Comparison of caries removal using Carisolv $^{\mathrm{TM}}$ or a conventional slow speed rotary instrument. Caries Res 1999; 33; 313.

26 Banerjee A, Watson T F, Kidd E A M. Carious dentine excavation using Carisolv ${ }^{\mathrm{TM}}$ gel: a quantitative autofluorescence assessment using scanning microscopy. Caries Res 1999; 33: 313 . 